\title{
Theoretical Delay Time Distributions
}

\author{
Gijs Nelemans, Silvia Toonen, and Madelon Bours \\ Dept. Astrophysics/IMAPP, Radboud University Nijmegen, P.O.. Box 9010, 6500 GL \\ Nijmegen, the Netherlands \\ email: nelemans@astro.ru.nl
}

\begin{abstract}
We briefly discuss the method of population synthesis to calculate theoretical delay time distributions of Type Ia supernova progenitors. We also compare the results of different research groups and conclude that, although one of the main differences in the results for single degenerate progenitors is the retention efficiency with which accreted hydrogen is added to the white dwarf core, this alone cannot explain all the differences.
\end{abstract}

Keywords. stars: supernovae, white dwarfs — binaries: close

\section{Introduction}

Given the uncertainties in the theoretical derivation of what the progenitors of Type Ia supernovae (SNe Ia) are, statistical methods can in principle be useful. Viable progenitor scenarios must not only potentially produce an SN Ia (i.e. an explosion that looks like observed SNe Ia), they must also occur often enough to explain the observed/inferred $\mathrm{SNe}$ Ia rate. In order to do so, an estimate needs to be made about the occurrence frequency of different types of binaries. This can be done using population synthesis techniques (e.g. Postnov and Yungelson 2006) as has been done by several groups for the different SN Ia progenitor scenarios (e.g. Yungelson and Livio 1998; Yungelson 2005; Han and Podsiadlowski 2004; Förster et al. 2006; Ruiter et al. 2009; Mennekens et al. 2010; Wang et al. 2009, 2010). In the following we will briefly discuss some of the important ingredients of population synthesis calculations, uncertainties and possible mitigating efforts before showing a comparison of results of different groups and an attempt to understand the differences.

\section{Population Synthesis}

The basics concept of population synthesis is simple: for a (large) sample of initial binaries (i.e. two Zero-Age Main Sequence stars orbiting each other with a certain orbital period) the subsequent evolution is determined. Folding those with assumed initial parameter (masses, periods) distributions and star formation histories, any binary population can be synthesized.

Population synthesis calculations typically evolve so many binaries that using a stellar evolution code to solve the structure equations explicitly is not feasible. Even if that would be the case, there are many binary evolution scenarios that cannot be computed with such codes anyway. Therefore people use rapid fits to evolutionary calculations or interpolations in pre-computed grids. This introduces uncertainties, although these are typically small compared to other uncertainties.

More problematic is determining the outcome of binary interactions. If one star in the binary fills its Roche lobe and starts to transfer matter to the companion, one first has to determine if this will lead to runaway mass transfer and the (likely?) start of a "common-envelope" phase. If not, the mass transfer is deemed "stable", but it still needs 


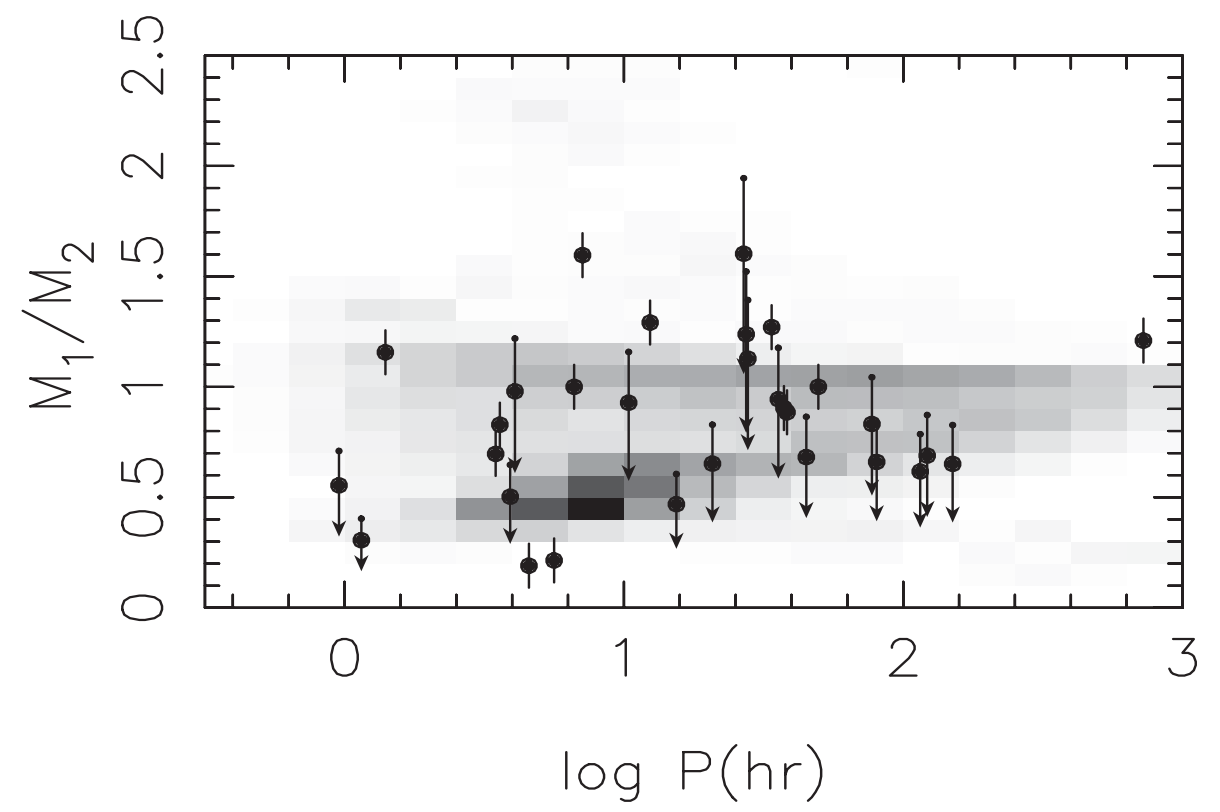

Figure 1. Period-mass distribution of the observed double white dwarfs compared to the results of the population synthesis of Toonen et al. in prep. The simulation matches the observations reasonably well.

to be determined how much of the transferred mass is accreted by the companion (likely depending on the speed at which the mass is transferred) and how the remaining mass leaves the system (i.e. how much angular momentum is lost along with the mass). That will have a large impact on the further evolution of the system.

In the context of SNe Ia, the two main progenitor scenarios are carbon-oxygen white dwarfs that either merge with other carbon-oxygen white dwarfs ["double degenerate" (DD) e.g. Webbink (1984)] or are accreting from a non-degenerate companion ["single degenerate" (SD) e.g. Whelan and Iben (1973)]. The most important uncertainties for population synthesis of these populations are the outcomes of mass transfer and, for the SD progenitors, the question of which white dwarf - companion star configurations lead to sufficient accretion to reach ignition conditions by approaching the Chandrasekhar mass. These uncertainties are sufficiently large that we cannot simply assume or hope that the prescriptions we use about these mass transfer phases in our population synthesis codes are right. Instead we have to use available observational data to calibrate the models.

\subsection{Comparison with Observations}

Comparison of the simulated populations with the observed population of (carbonoxygen) white dwarfs with non-degenerate companions has not be done yet to our knowledge. One of the reasons is that the observed population is severely biased by observational selection effects that need careful modeling before the observations can be compared to the population synthesis calculations. We are planning to do that in a forthcoming paper. The population of close double white dwarfs is much more homogeneous and we have compared the results of our population synthesis calculations with the observations to constrain the uncertain mass transfer phases (e.g. Nelemans et al. 2001, Toonen et al. in prep). In Figure 1 we show this comparison for our newest simulation of the double white dwarf population (Toonen et al. in prep., also these proceedings). 


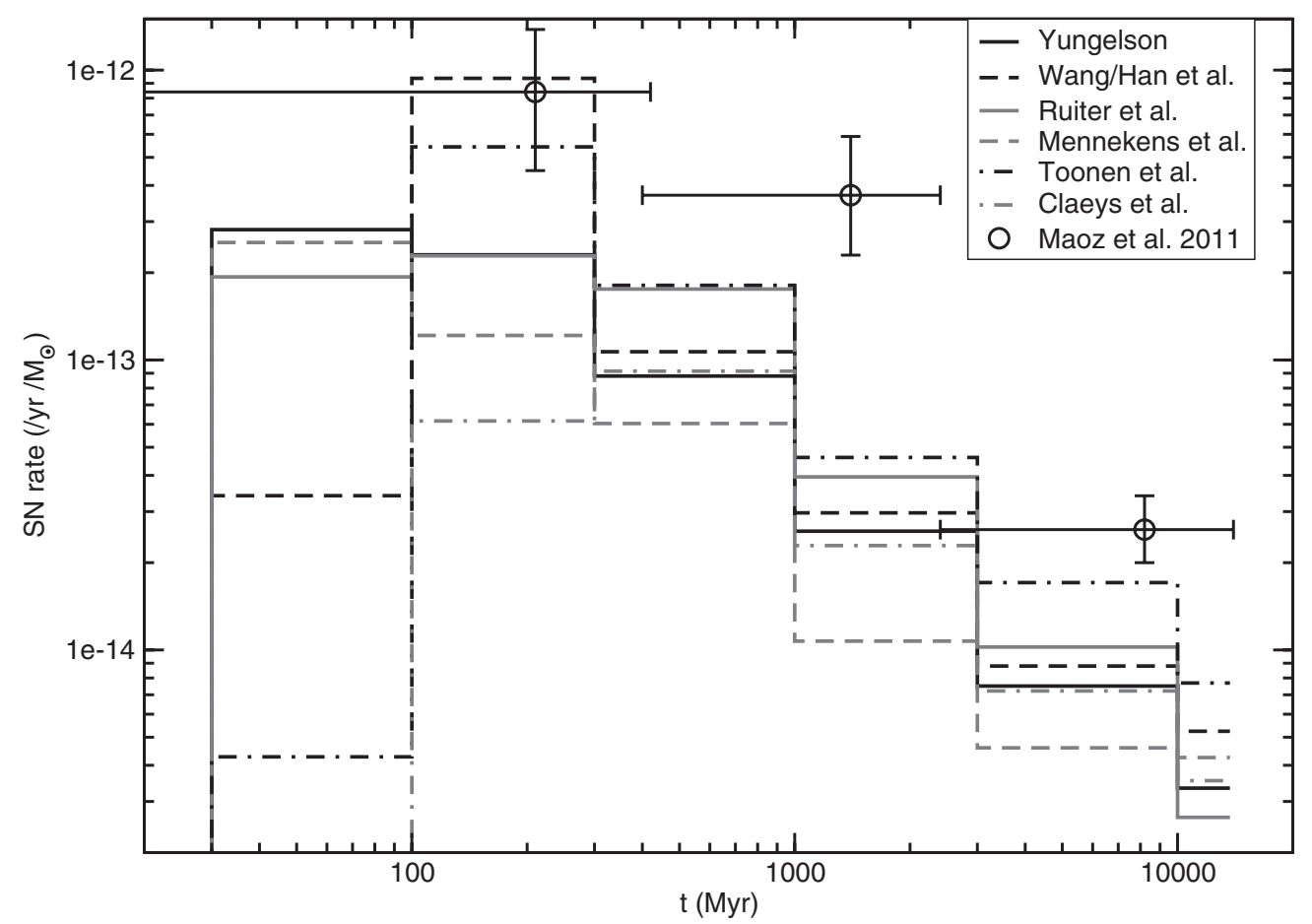

Figure 2. Rescaled DTDs for the DD scenario for the different groups.

There is a limit to the accuracy of this comparison when considering DD progenitor scenarios, because none of the observed systems are likely a double carbon-oxygen white dwarf with sufficient mass. However, the agreement with the other double white dwarfs does give some confidence in the simulation results.

\section{Delay Time Distributions of Different Groups}

Last year for the Lorentz center workshop "Observational signatures of Type Ia supernova progenitors", we asked a number of different research groups to give us their simulated data on Type Ia supernova progenitors so that we could do a comparison of the results of the different groups. All groups kindly accepted the invitation and were very helpful in clarifying any queries regarding their methods and normalization. We decided to make the comparison of the so-called "Delay Time Distribution" (DTD), which shows the SNe Ia rate per unit mass as a function of time since a hypothetical instantaneous starburst. For binary population synthesis calculations this is easy to compute - the only issue hampering direct comparison between groups is the normalization. This depends on the assumed initial mass function, the percentage of single stars, and the initial distribution of mass ratios and orbital periods. The groups involved are the following.

- Lev Yungelson (e.g. Yungelson 2010, 2005; Yungelson and Livio 2000).

- The Yunnan group (Han, Wang, etc.). See Wang et al. (2010); Han (2008); Han and Podsiadlowski (2004); Han (1998).

- The StarTrack code, Belczynski/Ruiter work (e.g. Ruiter et al. 2009; Belczynski et al. 2008).

- The Brussels group (see Mennekens et al. 2010).

- The Utrecht group, Claeys, Pols (e.g. Claeys, this proceedings). 


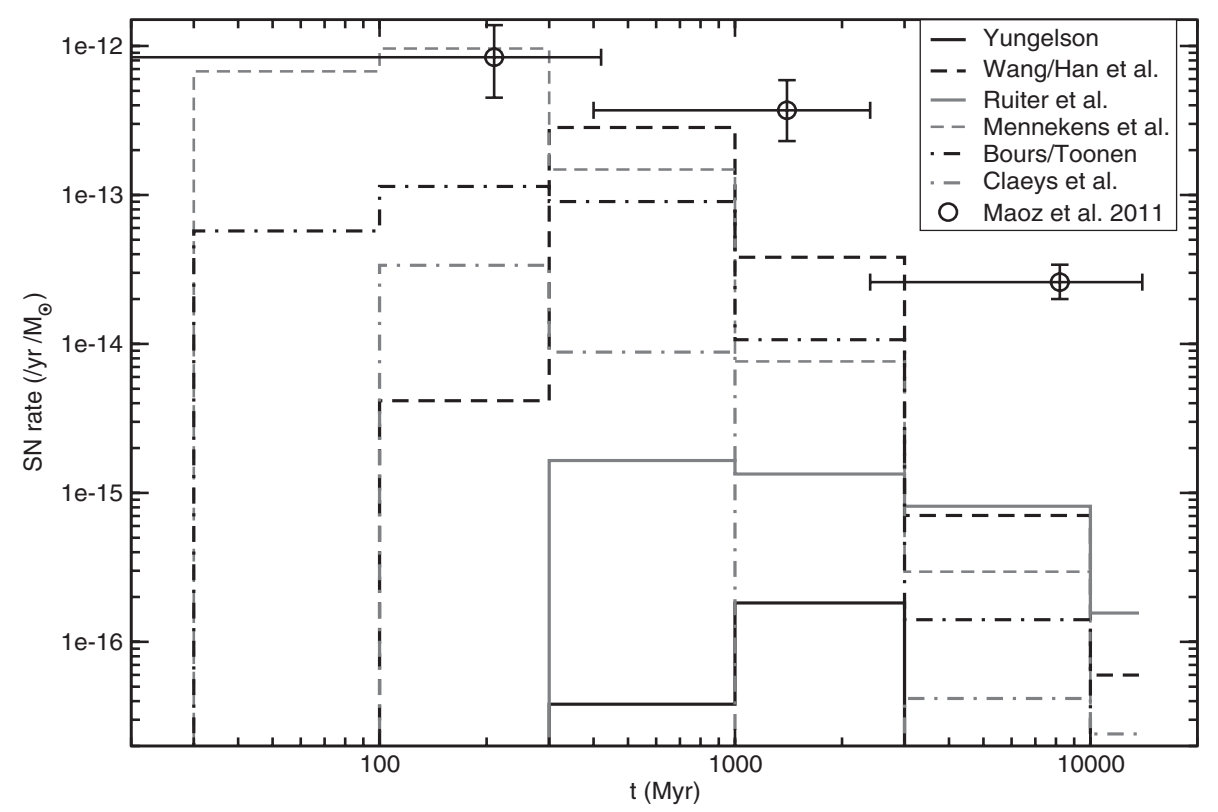

Figure 3. Rescaled DTDs for the SD scenario.

- Our SeBa code (Toonen et al., in prep; Nelemans et al. 2001; Portegies Zwart and Verbunt 1996).

For the comparison we rescaled the results (if needed) to the following assumptions: a Kroupa et al. (1993) IMF, flat mass ratio distribution, flat distribution in $\log P_{\text {orb }}$ and $50 \%$ binaries. The conversion factors for the different groups are given in Table 1 .

In Figures 2 and 3 we show the different groups' (binned) DTDs for the DD and SD scenarios, respectively. For the SD models we only considered systems with hydrogen rich donors, excluding helium rich donors (e.g. Wang et al. 2009). We also plot the inferred data points from Maoz et al. (2010). Most striking is that the double degenerate channel shows roughly the same shape in all the calculations, although with differences in the actual rates, but that the single degenerate DTDs are all over the place.

Because it is rather hard for the eye to integrate the area in a log-linear plot we show in Table 1 the integrated rates over a Hubble time (i.e. in total number of SNe Ia per $\mathrm{M}_{\odot}$ in a Hubble time), together with the inferred rate from Maoz et al. (2010). It is clear that all population synthesis calculations (with this normalization!) produce too few SNe Ia.

\section{Can We Understand the Differences?}

We investigated the most likely cause of the enormous discrepancy between the groups for the SD scenario: the actual conditions in which an accreting white dwarf can grow in mass and thus explode as an SN Ia (see Bours et al. in prep for more details). The different groups use different approaches: some use so-called retention efficiencies (the fraction of accreted hydrogen that in the end is added to the core of the white dwarf as carbon and oxygen), some use progenitor "islands" in parameter space (of orbital period, white dwarf and companion mass). We first calculated the SD DTD by using the islands as calculated by Hachisu et al. (2008), the same approach as taken by Mennekens et al. (2010). We also implemented retention efficiencies that are the basis of these islands, ones that are used by Ruiter et al. (2009) and ones that are used by Yungelson (2010) (see 
Table 1. Normalization factors and integrated SNe Ia rates in $10^{-4} \mathrm{M}_{\odot}^{-1}$ for the different groups and the different channels. For comparison the integrated rate inferred from the observations by Maoz et al. (2010) is also given.

\begin{tabular}{lrrr}
\hline Group & DD & SD & factor \\
\hline Yungelson & 2.4 & 0.006 & 0.8 \\
Wang/Han & 4.4 & 2.8 & 0.77 \\
Ruiter & 5.7 & 0.17 & $1 ?$ \\
Mennekens & 2.2 & 3.7 & 0.62 \\
Claeys & 1.9 & 0.13 & 1 \\
Toonen/Bours & 1.9 & 1.1 & 1 \\
\hline Observed & \multicolumn{3}{c}{$\mathbf{2 3}$} \\
\hline
\end{tabular}

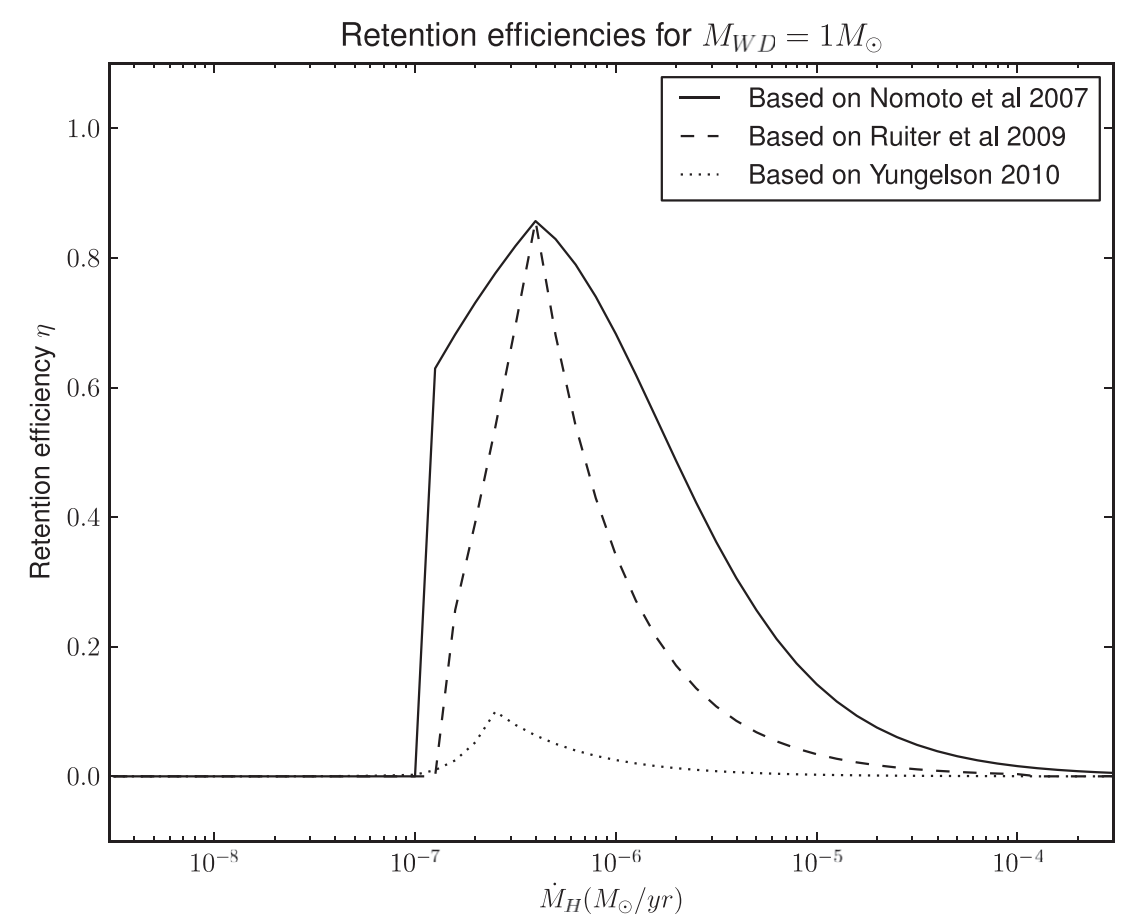

Figure 4. Retention efficiencies (i.e. efficiency with with accreted hydrogen is tuned into carbon and oxygen used by the different groups.

Figure 4). The latter two are based on calculations by Prialnik and Kovetz (1995); Iben and Tutukov (1996). The resulting DTDs of these calculations are shown in Figure 5. As can be seen, the different retention efficiencies do cause a large difference in the integrated rates (with no systems left with the "Yungelson" efficiencies), as is the case in the comparison in the previous section. However, the differences in the shape of the DTDs as found in the previous section are not reproduced in our modeling. This suggests that, apart from the retention efficiencies, other differences in the codes, most likely in the treatment of the mass transfer phases, contribute significantly to the uncertainties in the theoretical DTDs. 


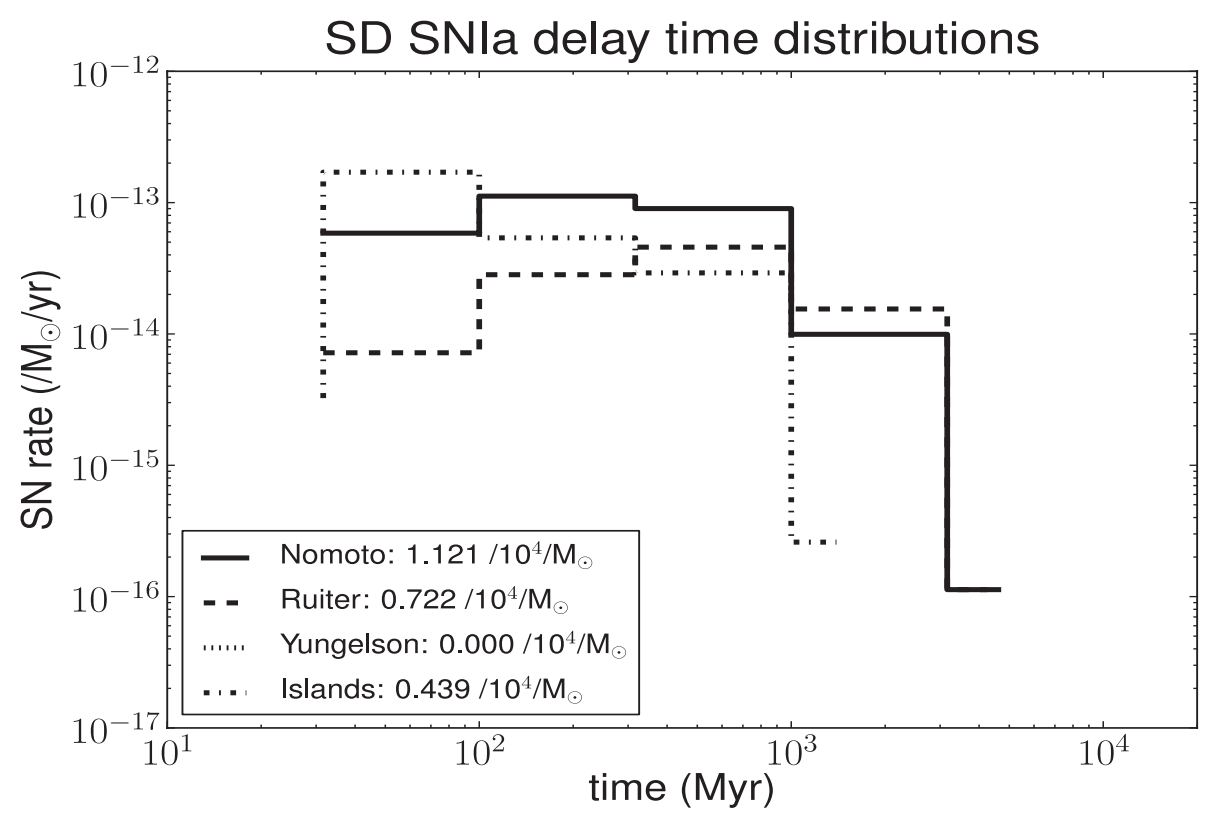

Figure 5. DTDs simulating the different approaches: "islands" for assuming the parameters that lead to SD SNe Ia to lie in the islands of Hachisu et al. (2008). The others are for the retention efficiencies shown in Figure 4 used by Yungelson (2010, 2005); Yungelson and Livio (2000); Ruiter et al. (2009); and one that should reproduce the islands.

\section{Conclusions}

We have shown that for the theoretical DTDs for the DD scenario there are (limited) observational tests that can be made to constrain the results. Interestingly, the DTDs of different research groups for the DD scenario agree reasonably well. For the SD scenario the DTDs differ wildly. This is partly due to the extreme uncertainty of the retention efficiency of accreted hydrogen on carbon-oxygen white dwarfs. However, a test in which this is varied while keeping the rest of the population synthesis code fixed, shows that this alone cannot explain the differences in the SD DTDs between the different research groups. Before the SD DTDs can be used to make any statements about the likelihood of this scenario, the retention efficiencies have to be determined better and the progenitor population has to be compared in detail to the local observed sample.

\section{Acknowledgments}

It is a great pleasure to thank our colleagues Lev Yungelson, Bo Wang, Zhanwen Han, Ashley Ruiter, Nicky Mennekens, and Joke Claeys for sharing their data with us and for numerous stimulating discussions.

\section{References}

Belczynski, K., Kalogera, V., Rasio, F. A., Taam, R. E., Zezas, A., Bulik, T., Maccarone, T. J., \& Ivanova, N. 2008, ApJS 174, 223

Förster, F., Wolf, C., Podsiadlowski, P., \& Han, Z. 2006, MNRAS 368, 1893

Hachisu, I., Kato, M., \& Nomoto, K. 2008, ApJ 683, L127

Han, Z. 1998, MNRAS 296, 1019

Han, Z. 2008, ApJ 677, L109 
Han, Z. \& Podsiadlowski, P. 2004, MNRAS 350, 1301

Iben, Jr., I. \& Tutukov, A. V. 1996, ApJS 105, 145

Kroupa, P., Tout, C. A., \& Gilmore, G. 1993, MNRAS 262, 545

Maoz, D., Mannucci, F., Li, W., Filippenko, A. V., Della Valle, M., \& Panagia, N. 2010, MNRAS 412, 1508

Mennekens, N., Vanbeveren, D., De Greve, J. P., \& De Donder, E. 2010, A $\& A$ 515, 89

Nelemans, G., Yungelson, L. R., Portegies Zwart, S. F., \& Verbunt, F. 2001, A\&A 365, 491

Portegies Zwart, S. F. \& Verbunt, F. 1996, A\&A 309, 179

Postnov, K. A. \& Yungelson, L. R. 2006, Living Reviews in Relativity 9, 6

Prialnik, D. \& Kovetz, A. 1995, ApJ 445, 789

Ruiter, A. J., Belczynski, K., \& Fryer, C. 2009, ApJ 699, 2026

Wang, B., Li, X., \& Han, Z. 2010, MNRAS 401, 2729

Wang, B., Meng, X., Chen, X., \& Han, Z. 2009, MNRAS 395, 847

Webbink, R. F. 1984, ApJ 277, 355

Whelan, J. \& Iben, I. J. 1973, ApJ 186, 1007

Yungelson, L. R. 2005, in E. M. Sion, S. Vennes, \& H. L. Shipman (eds.), White dwarfs: cosmological and galactic probes, Vol. 332 of Astrophysics and Space Science Library, Astrophysics and Space Science Library, pp 163-173

Yungelson, L. R. 2010, Astronomy Letters 36, 780

Yungelson, L. R. \& Livio, M. 1998, ApJ 497, 168

Yungelson, L. R. \& Livio, M. 2000, ApJ 528, 108 